\title{
Correction to: Genetically engineered cell lines for $\alpha 1$-antitrypsin expression
}

\author{
Qianqian Ji $@$ Caiping Guo $\cdot$ Chen Xie $\cdot$ Yingdan Wu $\cdot$ Pei Zhang • \\ Hui Li • Yongjun Lu
}

Published online: 23 July 2018

(C) Springer Nature B.V. 2018

Correction to: Biotechnol Lett (2017) 39:1471-1476

https://doi.org/10.1007/s10529-017-2391-5

In the original publication of the article, the Acknowledgement section was published incompletely. The complete Acknowledgement is given in this Correction.

Acknowledgements This work was supported by China Postdoctoral Science Foundation (Grant No. 2015M582465), and the innovative pharmaceutical engineering laboratory of Recombinant Plasma Protein in Shenzhen.

The original article can be found online at https://doi.org/10.1007/s10529-017-2391-5.

Q. Ji · Y. Lu

School of Life Sciences, Sun Yat-Sen University,

Guangzhou, People's Republic of China

C. Guo $\cdot$ C. Xie $\cdot$ Y. Wu $\cdot$ P. Zhang $\cdot$ H. Li

Shenzhen Weiguang Biological Products Co., Ltd.,

Shenzhen, People's Republic of China

Y. Lu (ه)

No. 135, Xin gang Xi Road, Guang Zhou 510275,

People's Republic of China

e-mail: luyj@mail.sysu.edu.cn 